\title{
Atendimento de urgência e emergência a clientes grávidas vítimas de trauma
}

\section{abdominal}

\author{
Urgency and emergency care for pregnant clients victims of abdominal trauma \\ Atención de urgencia y emergencia para clientas embarazadas víctimas de traumatismo abdominal
}

Recebido: 23/01/2022 | Revisado: 27/01/2022 | Aceito: 01/02/2022 | Publicado: 03/02/2022

José Ribeiro dos Santos

ORCID: https://orcid.org/0000-0002-1732-3143

Grupo Educacional Sequencial, Brasil

E-mail: zecasantos01@gmail.com

\begin{abstract}
Resumo
O trauma é uma contusão/lesão que envolve a troca de energia entre o meio ambiente e o corpo, é denominado também de "causas externas" e o abdômen é uma região vulnerável a sofrer traumas, o que pode comprometer órgãos vitais. O objetivo deste trabalho é analisar as produções científicas publicadas no período de 1995 á 2021 relacionado ao tema trauma abdominal em grávidas. Método: revisão bibliográfica de caráter descritivo qualitativo. Resultados. Os profissionais que atenderão essa vítima de trauma dever ter conhecimentos sobre os elementos fisiopatológicos que podem ocorrer nesta situação para prestar um melhor atendimento à gestante e diminuir a alta morbimortalidade materno-fetal. Conclusão: Muitos estudos acabam abordando os traumas em geral, entretanto há uma escassez de artigos nacionais atuais sobre o tema abordado, apesar das limitações, este estudo propõe fomentar essa busca e fornece insights para que estudos futuros possam incorporar ferramentas para dar suporte principalmente a equipe de enfermagem para prestar uma assistência de melhor qualidade a essa paciente.
\end{abstract}

Palavras-chave: Trauma abdominal; Gravidez; Atendimento de urgência e emergência.

\begin{abstract}
Trauma is a bruise/injury that involves the exchange of energy between the environment and the body, it is also called "external causes" and the abdomen is a region vulnerable to trauma, which can compromise vital organs. The objective of this work is to analyze the scientific productions published from 1995 to 2021 related to the topic of abdominal trauma in pregnant women. Method: bibliographical review of a qualitative descriptive character. Results. Professionals who will care for this trauma victim must have knowledge about the pathophysiological elements that can occur in this situation in order to provide better care to the pregnant woman and reduce the high maternal-fetal morbidity and mortality. Conclusion: Many studies end up addressing trauma in general, however there is a scarcity of current national articles on the topic discussed, abdominal trauma in pregnant women, despite the limitations, this study proposes to encourage this search and provides insights so that future studies can incorporate tools for support mainly the nursing staff to provide better quality care to this patient.
\end{abstract}

Keywords: Abdominal trauma; Pregnancy; Urgent and emergency care.

\section{Resumen}

El trauma es un hematoma / lesión que involucra el intercambio de energía entre el ambiente y el cuerpo, también llamado "causas externas" y el abdomen es una región vulnerable al trauma, que puede comprometer órganos vitales. El objetivo de este trabajo es analizar las producciones científicas publicadas entre 1995 y 2021 relacionadas con el tema del traumatismo abdominal en gestantes. Método: revisión bibliográfica de carácter descriptivo cualitativo. Resultados. Los profesionales que atenderán a esta víctima de trauma deben tener conocimiento sobre los elementos fisiopatológicos que pueden presentarse en esta situación, con el fin de brindar una mejor atención a la gestante y reducir la alta morbimortalidad materno-fetal. Conclusión: Muchos estudios terminan abordando el trauma en general, pero hay escasez de artículos nacionales actuales sobre el tema, trauma abdominal en gestantes, a pesar de las limitaciones, este estudio tiene como objetivo estimular esta búsqueda y brindar subsidios para que futuros estudios puedan incorporar herramientas de apoyo principalmente al personal de enfermería para brindar una atención de mejor calidad a este paciente.

Palabras clave: Trauma abdominal; Embarazo; Atención de urgencias y emergencias. 


\section{Introdução}

O ser humano está em constante risco de sofrer algum tipo de trauma durante a sua vida, as constantes evoluções tecnológicas e a invenção de diferentes instrumentos, por exemplo: a substituição do transporte de tração animal para os veículos motorizados, as armas brancas e projeteis de armas de fogo vêm aumentando ao longo da história da humanidade esse risco, até se tornando um problema de saúde pública a nível mundial. (Gómez, 2020).

Quando nos referirmos ao trauma a principal referência no atendimento á vítima politraumatizada é o ATLS (Advanced Trauma Life Support), um manual e instruções que sistematiza a sequência de atendimento ao ciente politraumatizado facilitando assim seu atendimento e melhorando de forma significativa a sobrevivência das vítimas e minimizando o aparecimento de novas lesões.

O abdômen é uma região vulnerável a sofrer traumas, o que poderá comprometer órgãos vitais, as lesões abdominais em mulheres grávidas é, sem dúvida, um problema para o médico assistente, se levarmos em consideração as complicações que podem causar a mãe e feto. (Labañino, et al. 2009).

Diversos estudos já demostraram que as anomalias por traumas atualmente representam um indicador importante de morbidade e mortalidade em todo o mundo, por essa razão deve-se focar no atendimento e tratamento rápido a cliente. $\mathrm{Na}$ abordagem a vítimas com traumas os músculos abdominais devem ser considerados fatores que podem potencialmente modificar o prognóstico do paciente. (Leite, et al. 2013)

Existem dois grandes grupos de mecanismos traumáticos: o fechado, mais prevalente, que inclui os acidentes de viação, quedas, entre outros, e o penetrante que abrange fundamentalmente as lesões por arma branca ou de fogo. pesquisas realizadas por (Parra-Romero, et al, 2019), mostra que os traumas por arma de fogo estão relacionados com as maiores lesões e, portanto, maior morbimortalidade, e que o controle destes representa uma política responsável pela saúde e segurança da população em geral.

As contusões/lesões que resulta de uma ação violenta, que envolve a troca de energia entre o meio ambiente e o corpo, é denominado também de "causas externas", o trauma na gravidez é um evento que pode levar a grande morbimortalidade. No Brasil, dados oficiais do Ministério da Saúde revelam que as chamadas "causas externas" se constituem a segunda causa de morte no país, superando as neoplasias, e precedidas, apenas, por doenças do aparelho cardiovascular.

Muitos dos aspectos de avaliação do trauma obstétrico são exclusivos da gravidez, embora a avaliação inicial e a reanimação devam ser sempre dirigidas pela mãe, quando a estabilidade materna é estabelecida, a avaliação vigilante do bemestar fetal torna-se necessária. (Mirza, et al, 2010)

É sabido que a avaliação de uma paciente grávida traumatizada no pronto-socorro requer uma rápida avaliação de suas lesões e imediata medidas terapêuticas, este atendimento deve ser dinâmico e multidisciplinar envolvendo as urgências e emergências: cirurgião, obstetra, neonatologista, anestesiologista e a equipe de enfermagem qualificada para uma assistência holística e humanizada a essa paciente.

Ao longo das últimas décadas o Brasil vem passando por um processo de transição epidemiológica com a modificação nos padrões de morte e morbidade. (Silveira et al, 2017).

Para minimizar isso, diversas estratégicas vêm sendo aplicadas e tem como objetivo assistir a gestante durante o ciclo da gravidez, desde as consultas de pré-natal (no mínimo sete), o parto, o puerpério até o segundo ano de vida do bebê. O trauma fetal direto é infrequente, já que o líquido amniótico amortece e dissipa as forças do trauma, (Júnior, et al, 1999).

A lesão craniana fetal mais comum resulta da fratura da pelve materna no final da gestação, quando a cabeça do feto está insinuada na apresentação fetal. O objetivo deste estudo é analisar as produções científicas publicadas no período de 1995 á 2021 relacionado ao tema trauma abdominal em grávidas. 


\section{Metodologia}

Trata-se de uma pesquisa de revisão bibliográfica de caráter descritivo qualitativo. O estudo é uma revisão sistemática que consiste em uma técnica científica objetiva, eficiente e reprodutível, a importância desta pesquisa está alicerçada no fato de que a revisão bibliográfica sistemática tem a finalidade de ratificar a efetividade de uma intervenção atualizada no processo de atendimento as pacientes grávidas vítimas de anomalias traumáticas

De acordo com a literatura, a revisão bibliográfica é definida como uma síntese de estudos primários que contém todos os elementos necessários claramente explicitados e que foi conduzida de forma clara e reprodutível, após a definição do tema foi realizada uma revisão sistemática da literatura científica em bases de dados virtuais relacionados ao atendimento de urgência e emergências a pacientes grávidas vítimas de lesões abdominal.

Tendo como base de dados: artigos científicos, livro, monografia, dissertações de mestrado e tese de doutorado em periódicos nacionais e internacionais: SCIELO (Scientifc Eletrinic Library Online), LILACS (Literatura Latino-Americana e do Caribe de Informação em Ciências da Saúde), MEDLINE (National Library of Medicine), PUBMED e Google Acadêmico. E as seguintes entidades: Ministério da Saúde e Colégio Brasileiro de Cirurgiões.

Pautando-se nas perspectivas teóricas adotadas, investigou-se os artigos inerentes ao tema abordado entre os anos de 1995 á 2021. Foram incluídos trabalhos na língua inglesa, portuguesa e espanhola que apresentavam combinações dos seguintes descritores: trauma abdominal, gravidez, atendimento de urgência e emergência.

Para os critérios de inclusão foram: textos disponibilizados em sua versão completa, escritos em português, inglês e espanhol com publicação a partir do ano de 1995 á 2021 e que atendiam os objetivos do estudo em questão. E para os critérios de exclusão: estudos publicados anterior ao ano de 1995, os estudos que não atendiam os objetivos do trabalho, estudos que não estavam disponíveis na íntegra, estudos em outros idiomas que não fossem Português, Inglês e Espanhol.

O levantamento dos textos ocorreu nas bases de dados de forma on-line entre os meses de setembro (25/09/2021) á dezembro (13/12/2021). A pesquisa realizada verificou 74 publicações potencialmente relevantes, procedeu-se as leituras e fichamentos de textos. Inicialmente procedeu-se a checagem dos títulos, autores e resumos, com objetivo de separar as publicações repetidas, a seguir foram esmiuçadas todas as publicações encontradas e selecionados os artigos inerentes ao assunto abordado os quais se adequavam aos objetivos do trabalho.

Os artigos foram selecionados para análise. Os dados obtidos serão armazenados em um programa de computador Word 2013 e serão discutidos na abordagem qualitativa.

\section{Resultados}

O trauma e suas eventuais complicações deverão ser abordados entre gestantes e os profissionais de saúde em todo o ciclo gravídico, minimizando os potenciais fatores de risco no lar ou até mesmo no trabalho da gestante. Pesquisas realizadas por (Costa, et al 2005), mostram que algumas medidas básicas como o uso de calçar sapatos adequados, sem saltos exagerados, confortáveis e antiderrapantes e o uso do corrimão é obrigatório em todas as escadas e devem estar em boas condições e sua fixação deve ser sempre revisada essas medidas são essências para minimizar os riscos de trauma na gestante. 
Quadro 1 - Apresentação dos principais artigos para o embasamento do estudo categorizados por: número, nomes dos autores, título do artigo, tipos de estudos, ano de publicação e revista vinculada. São Paulo, 2021.

\begin{tabular}{|c|c|c|c|c|c|}
\hline Número & Autores & Título do artigo & Tipo de estudo & Ano & Revista \\
\hline 1 & $\begin{array}{l}\text { Neil J. Murphy, Jeffreyd, } \\
\text { Quinlan. }\end{array}$ & $\begin{array}{l}\text { Trauma in } \begin{array}{l}\text { Pregnancy: } \\
\text { Assessment, } \\
\text { and Prevention. }\end{array}\end{array}$ & $\begin{array}{l}\text { Descritivo } \\
\text { qualitativo }\end{array}$ & 2014 & $\begin{array}{l}\text { American Academy } \\
\text { Family Physician. } 2014\end{array}$ \\
\hline 2 & $\begin{array}{l}\text { Leite, S. } \text { Gomes, AT. } \\
\text { Sousa, H }\end{array}$ & $\begin{array}{llr}\text { Lesão Visceral em } & \text { Trauma } \\
\text { Abdominal: Um } & \text { Estudo } \\
\text { Retrospetivo. } & & \\
\end{array}$ & $\begin{array}{l}\text { Análise retrospectiva } \\
\text { do processo clínico. }\end{array}$ & 2013 & Acta Médica Portuguesa \\
\hline 3 & $\begin{array}{l}\text { Figueroa, HM. Dahlke, JD. } \\
\text { Vrees, RA. Rouse, DJ. } \\
\text { MSPH }\end{array}$ & $\begin{array}{l}\text { Trauma in pregnancy: an } \\
\text { updated systematic review. }\end{array}$ & $\begin{array}{l}\text { Analítico } \\
\text { retrospectivo }\end{array}$ & 2013 & $\begin{array}{l}\text { Revista American Journal of } \\
\text { Obstetrics \& Gynecology }\end{array}$ \\
\hline 4 & $\begin{array}{l}\text { Ruezga, KLL. Gómez, JAJ. } \\
\text { González, LRR. Cruz, } \\
\text { MSS. Vigna, JJG. Barba, } \\
\text { TMT. }\end{array}$ & $\begin{array}{l}\text { Trauma abdominal cerrado y } \\
\text { penetrante con lesión a } \\
\text { órganos abdominales }\end{array}$ & $\begin{array}{l}\text { Descritivo e } \\
\text { retrospectivo }\end{array}$ & 2013 & Latinoamericana de Cirugía. \\
\hline 5 & $\begin{array}{l}\text { Hasbun, JH. Benitez, SS. } \\
\text { Cornejo, RR. Asencio, RC. } \\
\text { Navarro, JLA. Danilla, SE. }\end{array}$ & $\begin{array}{l}\text { Traumatismo materno grave y } \\
\text { cirugía múltiple con resultado } \\
\text { perinatal exitoso }\end{array}$ & $\begin{array}{l}\text { Estudo de caso/caso } \\
\text { clínico }\end{array}$ & 2011 & $\begin{array}{lcc}\text { Revista chilena } & \text { de } \\
\text { obstetrícia y ginecología } & \end{array}$ \\
\hline 6 & $\begin{array}{l}\text { Neil J. Murphy, Jeffreyd, } \\
\text { Quinlan }\end{array}$ & $\begin{array}{lll}\text { Pregnancy trauma: } & \text { a } \\
\text { systematic approach } & \end{array}$ & $\begin{array}{l}\text { Prospectivo analítico } \\
\text { comparativo }\end{array}$ & 2010 & $\begin{array}{lll}\text { American } & \text { Journal of } \\
\text { Perinatology } & \end{array}$ \\
\hline 7 & $\begin{array}{l}\text { Labañino, W. } \text { L., } \\
\text { Muguercia, R. E., Alonso, } \\
\text { A. C., Nuñez, E. S., } \\
\text { Terrero, I. R., \& Reyes, W. } \\
\text { J. }\end{array}$ & $\begin{array}{l}\text { Trauma abdominal en el } \\
\text { embarazo. Actualización }\end{array}$ & Revisão bibliográfica & 2009 & $\begin{array}{l}\text { Revista de Información } \\
\text { Científica, }\end{array}$ \\
\hline 8 & $\begin{array}{l}\text { Fraga GP, Mantovani M, } \\
\text { Mesquita AC Soares AB, } \\
\text { Passini Júnior R }\end{array}$ & $\begin{array}{lll}\text { Trauma } & \text { abdominal } & \text { em } \\
\text { grávidas. } & & \end{array}$ & Análise retrospectiva & 2005 & $\begin{array}{l}\text { RevistaBrasileira de } \\
\text { Ginecologia e Obstetricia }\end{array}$ \\
\hline 9 & $\begin{array}{l}\text { Júnior, GAP. Júnior, LFH. } \\
\text { Atique, JMC. Nakamura, } \\
\text { EJ. Filho, AB. Andrade, } \\
\text { JID }\end{array}$ & $\begin{array}{lll}\begin{array}{l}\text { Atendimento } \\
\text { traumatizada }\end{array} & \text { á } & \text { gestante } \\
\end{array}$ & $\begin{array}{c}\text { Abordagem } \\
\text { descritiva qualitativa }\end{array}$ & 1999 & $\begin{array}{l}\text { Revista Medicina, Ribeirão } \\
\text { Preto }\end{array}$ \\
\hline
\end{tabular}

Fonte: Autores.

Do total de 22 artigos selecionados para compor esse estudo, 15 artigos foram escritos por profissionais médicos, representando um percentual de (68,19\%). 3 artigos foram escritos por profissionais enfermeiros (13,63\%). E 4 artigos escritos por estudantes de medicina com seus professores, que correspondem a (18,18\%). Quanto a abordagem: foram 4 artigos analíticos retrospectivos, 1 artigo analítico descritivo de coorte transversal, 1 artigo com abordagem retrospectivo, observacional e descritivo, 2 artigos observacional, prospectivo analítico comparativo, 5 artigos de revisão bibliográfica, 1 estudo de caso, 2 artigos analítico descritivo prospectivo com coleta de dados, 1 estudo de caso observação sistemática com coleta de dados, 3 artigos observacional descritivo longitudinal com abordagem prospectiva, 1 artigo de revisão sistemática de literatura, e 1 artigo com abordagem descritivo qualitativo.

De acordo com as pesquisas, realizadas por (Mirza, et al, 2010), as principais causas de trauma obstétrico são acidentes com veículos motorizados, quedas, agressões e disparos de arma de fogo, as lesões subsequentes são classificadas como lesões contusas, fraturas pélvicas ou trauma penetrante.

A literatura aponta que os acidentes com veículos motorizados no geral, $2 \%$ das mulheres grávidas estão envolvidas em acidente de veículo motorizado durante a gravidez. Os impactos gerados pelas causas externas têm repercussão em diversos setores da sociedade, mas é o setor saúde que recebe seu maior impacto, pela pressão que exercem suas vítimas sobre os serviços de emergência, recuperação e reabilitação. (Silveira, et al, 2017). 


\subsection{Anatomia Abdominal}

O abdômen é delimitado pelo diafragma, pela pelve e o peritônio que é uma membrana serosa transparente, as regiões do abdome são divisões teóricas utilizadas por clínicos para auxiliar na localização, identificação e diagnóstico dos sintomas apresentados pelos pacientes.

Existem duas formas principais de categorização, a primeira mais simples e observada pela divisão do abdome em quatro quadrantes, e o segundo método, que divide o abdome em nove segmentos. Os 4 quadrantes são: quadrante superior direito e esquerdo e quadrante inferior direito e esquerdo. Já a divisão dos nove seguimentos são: hipocôndrio direito, epigástrica, hipocôndrio esquerdo, flanco direito, mesogástrio, flanco esquerdo, fossa ilíaca direita, hipogástrica, fossa ilíaca esquerda. (Berg, et al, 2014).

O peritônio é formado por duas lâminas contínuas: peritônio parietal e visceral, essas estruturas podem ser reconhecidas as quais são suscetíveis a danos durante trauma abdominal: o mesentério, que conecta um órgão intraperitoneal e a parede corporal, proporcionando meio de fixação e comunicação vascular e nervosa; a omento, que cobre os órgãos; e o ligamento peritoneal, que conecta um órgão a outro ou com a parede abdominal. (Gómez, 2020).

Os órgãos abdominais e pélvicos são: estômago, fígado, vesícula biliar, pâncreas e ductos biliares, baço, intestinos delgado e grosso, rins, glândulas adrenais e os vasos abdominais, que são ramificações da aorta abdominal. A bexiga urinária, (útero, tuba uterina e ovários em mulheres).

\subsection{Epidemiologia do trauma: classificação do trauma: fechado e penetrante}

O mecanismo de trauma: agente de lesão, regiões anatômicas afetadas, o tempo de atendimento, estado hemodinâmico e neurológico da paciente são eventos que poderão levar a grande morbimortalidade por essa razão deve-se fazer uma abordagem sistematizada no atendimento, que inclua sequência hierarquizada de prioridades e seja facilmente aplicada e reavaliada.

Trauma abdominal aberto - Por armas brancas (penetrante ou perfurante), trauma abdominal fechado são classificados em: leve, moderado e severo/grave, onde o paciente poderá necessitar de uma atenção maior. Nos traumas leves, geralmente limitados a abrasões, lacerações e pequenos hematomas da parede abdominal sem lesão das vísceras intra-abdominais

No trauma moderado são aqueles que vêm acompanhados de contusão ou hematoma nas vísceras síndrome intraabdominal não hemorrágica geralmente resolvem com tratamento médico sem grandes consequências para a mãe, mas pode causar complicações como: descolamento prematuro da placenta ou parto prematuro de risco elevado para a saúde do feto. (Labañino, et al, 2009).

As lesões traumáticas geralmente abrangem um amplo espectro clínico e radiológico e são identificadas em aproximadamente 9\% dos clientes apresentando lesões fechado. (Scott, et al, 2021). Pesquisas mostram que o trauma severo é aquele que são acompanhados por lesões abdominais com hemorragias e que também está associada a lesões de outras órgãos ou sistemas fora da cavidade abdominal. Exemplo: fraturas de ossos longos, traumas torácicos e / ou cerebrais (os últimos estão associados a uma maior taxa de mortalidade materna).

Os profissionais que atenderão essa vítima de trauma dever ter conhecimentos sobre os elementos fisiopatológicos que podem ocorrer nesta situação para prestar um melhor atendimento à gestante e diminuir alta morbimortalidade materno-fetal, os mecanismos que estão na origem de uma lesão traumática são importantes na abordagem diagnóstica e terapêutica de cada caso. (Leite, et al, 2013).

As lesões traumáticas mais comuns são colisões de veículos motorizados, agressões, quedas e armas de fogo. (Neil et al, 2014). O tipo de tratamento dependerá da gravidade das lesões sofridas e o estado hemodinâmico da paciente, o 
socorrista/equipe de saúde deverá estabelecer prioridades de tratamento, atentando-se para o nível de consciência e as alterações físicas e clinicas da cliente.

Para a redução da morbimortalidade por acidentes e violência, foi criado a Política Nacional instituída pela portaria $n^{\circ}$ 737/GM em 16 de maio de 2001, que prioriza medidas preventivas contra acidentes e violência, além da garantia de tratamento às vítimas destes eventos. Estas intervenções devem ser conjuntas entre profissionais de saúde, o setor público a sociedade civil, para que sejam eficazes na conscientização sobre os agravos ocasionados pelo infortúnio. (Brasil- Ministério da Saúde, 2005). Esse enfoque baseia-se no fato de que quanto mais se investe na prevenção primária, menor é o custo no atendimento às vítimas e maior o impacto e a abrangência na proteção da população.

\subsection{Estabelecendo um diagnóstico e possível tratamento para a cliente}

Os estudos radiológicos simples são protocolares e devem se realizar já que neles podemos visualizar ruptura de hemidiafragma, pneumoperitônio ou fraturas da caixa torácica ou coluna lombar que nos leva a suspeita de lesões. No trauma abdominal leve com a vítima hemodinamicamente estável e que não requer uma cirurgia de laparotomia de imediata, deve-se fazer um exame físico completo e uma avaliação detalhada com exames de imagem, principalmente com tomografia, pois dá uma visão mais específica sobre o órgão intra-abdominal afetado e auxilia na tomada de decisões. (Gómez, 2020).

O exame físico continua sendo o principal meio diagnóstico, além do exame físico detalhado o profissional médico deverá solicitar exames laboratoriais complementares e procedimentos. E depois, seguir com a avaliação clínica da paciente para verificar quais órgãos foram afetados, estabelecendo assim uma conduta. Normalmente as lesões intestinais exigem intervenção cirúrgica para evitar peritonite e choque séptico.

Na literatura consultada, joga luz ao estabelecer condutas apropriadas as diferentes situações de trauma: Trauma abdominal fechado: Situação 1: paciente instável com lesões limitadas ao abdômen. Conduta: laparotomia exploradora. Situação 2: paciente muito grave, instável, com diversos ferimentos e sem que se saiba se a lesão causadora da instabilidade hemodinâmica está no abdômen. Conduta: lavado peritoneal ou ultrassom FAST. Situação 3: paciente com lesões múltiplas, estável, cuja lesão abdominal se existir não constitui perigo de vida imediato. Conduta: TC abdômen para detectar lesão de vísceras sólidas e ou líquido livre na cavidade.

Situação 4: Paciente estável com líquido livre na cavidade abdominal e lesão de víscera sólida (fígado ou baço) demonstrado pela TC de abdômen. Conduta: pode-se tentar o tratamento conservador. Situação 5: Paciente estável com líquido livre na cavidade e sem lesões de fígado ou baço na TC de abdômen. Conduta: laparotomia exploradora pois o líquido pode ser lesão de víscera oca. (Markogiannakis, et al, 2006).

\section{Discussão}

A Associação Americana para a Cirurgia do Trauma (AAST) é a maior de todas organizações de trauma, propuseram uma escala de lesão de órgão para cirurgia de trauma, a escala é classificada de 1 a 6 para cada órgão, 1 é a lesão menos grave e 5 a lesão mais grave. do qual o paciente pode sobreviver. Sendo grau 6, aqueles órgãos que não podem ser salvos. Essa escala é usada para a tomada de decisão no momento da laparotomia exploratória, incluindo controle de hemorragia, identificação de lesões, controle de contaminação e reconstrução. (Gómez 2018). De acordo com pesquisas realizadas por (Figueroa, et al, 2013) Estima-se que o trauma complica aproximadamente 1 em 12 gestações e é a principal causa não obstrutiva de morte materna e com implicações fetais aumenta a incidência de aborto espontâneo prematuro, ruptura prematura de membranas, parto prematuro (PTB), ruptura uterina, parto cesáreo.

No trauma se faz necessário o monitoramento cardíaco fetal contínuo, ultrassonografia, tomografia computadorizada, lavagem peritoneal aberta e / ou laparotomia exploradora podem ser indicados em um caso de trauma obstétrico, para isso o 
profissional médico deverá realizar uma avaliação minuciosa para tomar uma conduta assertiva. Tendo em vista o impacto significativo do trauma na gestante e no feto, estratégias preventivas são fundamentais. (Fraga, et al, 2005).

Trauma é uma das principais causas de morbidade e mortalidade em todo o mundo e continua sendo um grande problema de saúde pública entre todos os países. Foi estimado pelo ano 2020, 8,1 milhões de pessoas morreriam anualmente como resultado de lesões, a região do abdome representa 25\% (Bowles, et al, 2021)

Pesquisas realizadas por Fraga, et al 2005, mostra que os índices de trauma não foram fatores preditivos de prognostico para a mãe ou feto, os autores sugerem incorporação de ferramentas para o desenvolvimento de sistemas que possa avaliar a estimativa de prognostico materno fetal.

Já para (Labañino, et al, 2009), os autores sugerem que a implementação obrigatória de airbags para motoristas e passageiros do banco traseiro é um mecanismo importante de proteção e que as mulheres grávidas devem ser encorajadas e instruídas sobre o uso de cintos de segurança que já provou diminuir a mortalidade fetal, seu uso demonstrou reduzir ambos quantidade como a gravidade das lesões e é considerada como diminuindo em $43 \%$ de risco de morte e $43-52 \%$ de risco de lesão importante.

Acidente é entendido como o evento não intencional e evitável, causador de lesões físicas e/ou emocionais no âmbito doméstico ou nos outros ambientes sociais, como o do trabalho, do trânsito, da escola, de esportes e o de lazer. Pesquisas realizadas por (Figueroa, et al, 2013), relata que quando o feto for viável o monitoramento fetal deverá ocorrer o mais rápido possível e que a gravidez não deve levar a um subdiagnóstico ou subtratamento, os autores revelam ainda que o fato de houver fraturas pélvicas não necessariamente impedem o parto vaginal. E que se a lavagem peritoneal for indicada, a técnica aberta é preferível, colocação de um cateter de Foley e sonda nasogástrica.

Para um atendimento adequado a grávida traumatizada é necessário considerar as alterações anatômicas e fisiológicas da gravidez que se tornam mais evidente a partir do terceiro mês de gestação. As modificações fisiológicas da gravidez interferem na avaliação do estado hemodinâmico da paciente em pode provocar demora no estabelecimento de medidas corretivas ou diagnostica. (Fraga, et al, 2005).

Na gestante além do exame físico completo, o monitoramento da frequência cárdica fetal e exames radiológicos, os autores (Júnior et al, 1999), sugere que o exame ginecológico completo tembem deverá ser realizado e que a presença de contrações uterinas sugere parto prematuro e se essas contrações forem associadas a sangramento vaginal sugere um deslocamento prematuro de placenta.

Pesquisas realizadas por (Ruezga, et al,2013) mostram que na laparoscopia diagnostica existem muitas limitações como a incapacidade de viajar pelo intestino, diagnosticar lesões retroperitoneais, exposição de órgãos em planos profundos e a estimativa exata para indicação da laparotomia.

De acordo com (Costa, et al, 20005), a cesariana post-mortem está plenamente justificada diante de um feto vivo e viável intra-útero. Quanto menor o tempo entre a parada cardiorrespiratória materna e a cesárea, melhores são os resultados perinatais, indo de $98 \%$ de sobrevida se a intervenção é efetuada até 5 minutos após a morte materna a $25 \%$ se esta ocorre entre 26 e 35 minutos.

Nesta era de modalidades diagnósticas modernas inúmeras ferramentas de diagnóstico são acessíveis, entretanto só ajudam na confirmação da suspeita clínica, a decisão quanto a natureza do procedimento a ser realizado é do profissional médico em uma situação de urgência e emergência,

Várias investigações de modalidades como avaliação focada com ultrassonografia para trauma (FAST), lavagem peritoneal diagnóstica (DPL) e tomografia computadorizada com contraste aprimorado (CECT) abdômen e pélvis poderão se aplicáveis dependendo do estado hemodinâmico da vítima, se a paciente se encontra instável em choque hipovolêmico 
profundo não responde ao desafio de fluidos e transfusão de sangue exige exploração cirúrgica imediata para controlar a hemorragia.

Pesquisas apontadas por Costa, et al, 2005, mostram que em um acidente a gestante pode perder em torno de 30-35\% do seu volume circulante antes de apresentar taquicardia significativa, hipotensão arterial ou outras alterações volêmicas por exemplo: (choque hipovolêmico). O trauma físico ocorre em aproximadamente de 6 a7\% e nem sempre há a necessidade de hospitalização e pesquisa aponta que houve predomínio dos traumas penetrantes por projéteis de armas de fogo.

De acordo com (Gonzalo, 2017 \& Parra-Romero, et al, 2018) no trauma abdominal há associação entre os tipos de traumas [aberto e fechado] e o gênero, os traumas abertos com maiores índices de ocorrências e que no gênero masculino a probabilidade de trauma abdominal a faixa etária é de 15 a 40 anos, já no gênero feminino a idade é de 21 a 30 anos. Os órgãos mais afetados no trauma aberto foi o intestino delgado e no trauma fechado o fígado. Estudos retrospectivos realizados por (Costa, et al, 2005), mostra que os pacientes submetidos a laparotomia devido ao trauma foi realiza na população masculina e feminino. Entre a população feminina o número de cirurgias em mulheres grávidas pós trauma corresponde a 5,3\% e 6,3\% das intervenções em mulheres em idade reprodutiva.

A notável diferença entre os dois sexos, com predominância de 4:1 do sexo masculino sobre o feminino, tem sido explicada pela maior exposição de homens a armas de fogo e o maior consumo de substâncias como álcool e drogas ilícitas, que os levaria a ter comportamentos mais agressivos e acidentes por descuido (Parra-Romero, et al, 2018). Uma cesárea de urgência diante de um quadro de parada cardiorrespiratória deverá ser realizado as manobras de ressuscitação cardiopulmonar (RCP) de forma contínua. Após 5 minutos de PCR o socorrista deverá avaliar o status materno: idade gestacional, viabilidade fetal condições favoráveis para reanimação do feto.

De acordo com o cenário o profissional poderá tomar uma conduta assertiva ao prestar atendimento a vítima gestante de trauma, o fluxograma abaixo mostra de forma sistematizada as ações/ medidas que o socorrista deverá executar de acordo com o quadro apresentado pela cliente. 
Figura 1 - Algoritmo de gestão de atendimento sistematizado a gestantes vítimas de traumas.

Avaliar status materno $\quad$ - Parada Cardíaca
-Perda de vias aéreas/ parada respiratória
$-\mathrm{PA}<80 / 40 \mathrm{~mm} \mathrm{Hg} \mathrm{ou} \mathrm{FC}<50 \mathrm{ou}>140 \mathrm{bpm}$
Se o feto for viável, FHR $<110 \mathrm{ou}>160 \mathrm{bpm}$

PRESENTE

\section{Suporte avançado de vida}

Controle das vias aéreas / coluna cervical

Respirando

Circulação

Incapacidade

Exposição

Consulta com equipe de trauma; notificar NICU

Suplementação com oxigênio $0_{2}$

Desloque o útero para a esquerda se GA> 20 semanas

Acesso IV (2 linhas periféricas)

Laboratórios: $\mathrm{CBC}$, perfil de coagulação, tipo e tela; KB se $\mathrm{Rh}(-)$, digite \& cruze

Feto viável: monitoramento contínuo de FCF

Feto previsível: monitorar frequência cardíaca fetal via Doppler

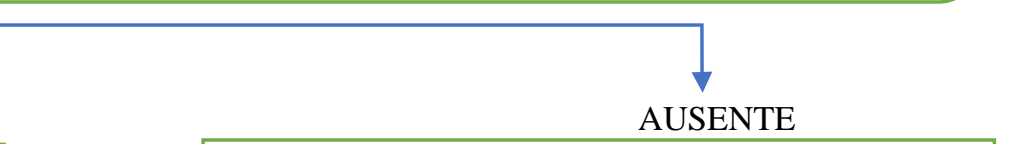

Lesão materna maior que menor hematomas, lacerações ou contusões

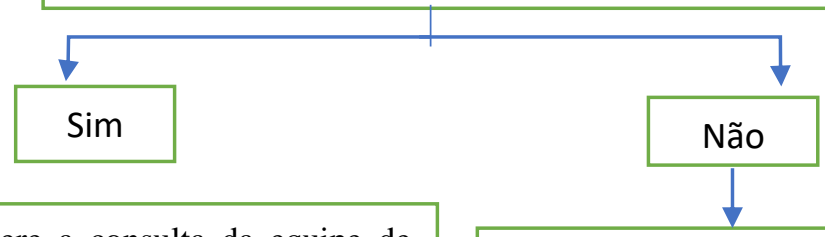

Breve avaliação fetal trauma

Acesso IV

Laboratórios: CBC, perfil de coagulação, tipo e tela; KB se Rh (-) Feto viável: monitoramento fetal por 4 horas

Contrações $<6$ / hora, considerar alta

Contrações $\geq 6$ / hora considerar admissão

Feto previsível: monitorar frequência cardíaca fetal via Doppler
Nenhuma avaliação de laboratório necessária

Sem imagem radiológica requeridas

\section{Assim que o paciente estiver estável Ultrassom fetal +/- Perfil Biofísico}

Considere outros exames: gasometria arterial, exames de urina, (exame de toxicologia da urina)

Avaliação radiológica / lavagem peritoneal/FAST e USG (se indicado)

\begin{tabular}{|c|c|c|c|c|c|}
\hline$\downarrow$ & & & & & $\downarrow$ \\
\hline $\begin{array}{l}\text { MVA } \\
\text { Determinar se } \\
\text { paciente } \\
\text { estava usando } \\
\text { o cinto de } \\
\text { segurança. }\end{array}$ & \begin{tabular}{l}
\multicolumn{1}{c}{$\begin{array}{c}\text { Escorregões / } \\
\text { quedas }\end{array}$} \\
Avaliação \\
abdominal, trauma \\
nas extremidades \\
para fratura /dano \\
ao ligamento
\end{tabular} & $\begin{array}{l}\text { Queimaduras } \\
\text { Agressivo-fluido } \\
\text { ressuscitação } \\
\text { Consider delivery } \\
\text { if burn area >50\% }\end{array}$ & 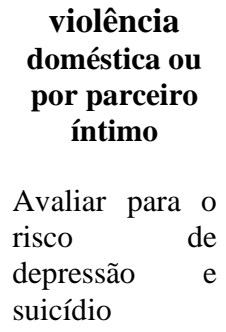 & $\begin{array}{l}\text { Trauma penetrante } \\
\text { O nível de entrada } \\
\text { afeta determinado } \\
\text { órgão. } \\
\text { O útero gravídico pode } \\
\text { proteger lesão viceral }\end{array}$ & $\begin{array}{l}\text { Exposição tóxica } \\
\text { o agente tóxico/ IG } \\
\text { guias de exposição } \\
\text { terapia materna e } \\
\text { aconselhamento }\end{array}$ \\
\hline
\end{tabular}

Algoritmo proposto para avaliação e tratamento do trauma na grávida. (adaptação e tradução parcial o autor). BP, blood pressure; CBC, complete blood cell count; Ctxs, contractions; DV, domestic violence; FAST, focused assessment with sonography for trauma; FHR, fetal heart rate; GA, gestational age; HR, heart rate; IPV, intimate partner violence; ISS, Injury Severity Score; IV, intravenous; KB, Kleihauer-Betke; MVA, motor vehicle accident; NICU, neonatal intensive care unit; O2, oxygen; U/S, ultrasound. FCFrequência Cardíaca; FRH- frequência cardíaca fetal; NICU (UTI) Neonatal; GA- (ID) Idade Gestacional; IV- Via Intravenosa; CBC-Contagem completa de células sanguíneas (Hemograma completo); FAST- Avaliação focada com ultrassonografia para trauma; MVA- Acidente com veículo motorizado; USG-ultrassonografia. Fonte: Mendez-Figueroa.

Trauma in pregnancy. Am J Obstet Gynecol 2013.pag. 6. 
Avaliação focada com ultrassonografia para trauma é confiável durante a gravidez e a cesariana pode ser apropriado no cenário de iminente morte materna ou após 4 min de executado corretamente, mas sem sucesso ressuscitação cardiopulmonar. A abordagem ao trauma visa cada vez mais uma avaliação focada no diagnostico rápido com métodos menos invasivo e se possível um tratamento não operatório. (Leite, et al, 2013). Pesquisas mostram que para o atendimento dessa paciente além de um atendimento rápido e eficaz é preciso uma equipe multidisciplinar para assistir essa cliente

Para evitar complicações decorrente de infecções, ultimamente vem se optando por um tratamento não cirúrgico no trauma abdominal fechado (Mestieri, et al). Para essa abordagem é preciso uma avaliação, o uso crescente do TNO é baseado nas baixas taxas de insucessos relatados em estudos retrospectivos.

\section{5- Conclusão}

Foi possível observar que o trauma durante a gravidez está associado ao aumento de risco de aborto, parto prematuro, deslocamento prematuro de placenta, ruptura uterina e hemorragia feto-materna e para ter um resultado bem sucedido é preciso uma intervenção medica e cuidados contínuos e abrangentes nas tomadas de decisões do tratamento, médico e cirúrgico e que essas medidas envolvem toda uma equipe multidisciplinar.

Muitos estudos acabam relatando os traumas em geral, entretanto há uma escassez de artigos nacionais atuais sobre o tema abordado, apesar das limitações, este estudo busca fomentar essa busca e fornece insights para que estudos futuros possam incorporar ferramentas para melhoria do atendimento a essas pacientes contribuindo assim, com o desenvolvimento e fortalecendo e instrumentalizando a equipe que irão atuar no atendimento ao trauma.

Embora seja um tema relevante, observa-se que é pouco explorado em parte na cultura nacional talvez seja porque a sociedade ainda atribui a mulher grávida a probabilidade de um risco baixo de sofrer um trauma abdominal já que durante a gravidez ficam menos expostas a agentes físicos, químicos e radiativos e por Lei são afastadas determinado tempo do seu trabalho durante a gestação, entretanto isso não isenta a mulher grávida de sofrer um trauma seja no trabalho, no itinerário ou até mesmo em sua casa. Algumas sugestões de estudos futuros para que possa corroborar a pesquisa: *Índices de sobrevivência de mulheres grávidas após trauma severo com reanimação cardiopulmonar. *indicadores da qualidade do atendimento a mulheres gestantes vítimas de violências *estudos comparativos: taxa de sobrevivência de mulheres gravidas vitimas de trauma abdominal sem reanimação e com reanimação.

\section{Referências}

Berg, R. J. et al (2014). The contemporary management of penetrating splenic injury. Injury, 45, (9), 1394-400. Doi:https://doi.org/10.1016/j.injury.2014.04.025

“Brasil. (2005). Ministério da Saúde. Secretaria de Vigilância em Saúde Departamento de Análise de Situação de Saúde. Política Nacional de Redução da

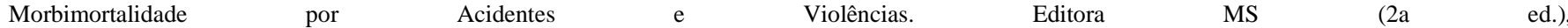
https://bvsms.saude.gov.br/bvs/publicacoes/politica_reducao_morbimortalidade_acidentes_2ed.pdf

Brasil. (2013). Colégio Brasileiro de Cirurgiões-Trauma-terceiro programa de auto-avaliação em cirurgia. Editora Diagraphic. https://cbc.org.br/wpcontent/uploads/2013/05/Ano1-II.Trauma.pdf

Bowles, M., \& Weller, J. (2021). Abdominal and pelvic vascular trauma in Queensland, Australia: institutional experience at a level one trauma centre. International Surgery Journal, 8(4), 1075-1079.

Deheza, G. C. (2017). Análisis comparativo entre indicadores de trauma abdominal, em el pronóstico de morbimortalidad. Revista Médico-Científica "Luz y Vida". 8(1), 14-19.

Mendez-Figueroa, H., Dahlke, J. D., Vrees, R. A., \& Rouse, D. J. (2013). Trauma in pregnancy: an updated systematic review. American journal of obstetrics and gynecology, 209(1), 1-10. doi: 10.1016/j.ajog.2013.01.021.

Fraga, G. P., Mantovani, M., Mesquita, A. C., Soares, A. B., \& Passini Júnior, R. (2005). Trauma abdominal em grávidas. Revista Brasileira de Ginecologia e Obstetrícia, 27, 541-547. 
Research, Society and Development, v. 11, n. 2, e48811226067, 2022

(CC BY 4.0) | ISSN 2525-3409 | DOI: http://dx.doi.org/10.33448/rsd-v11i2.26067

Hasbun, J., Benitez, S., Cornejo, R., Asencio, R., Navarro, J. L., \& Danilla, S. (2011). Traumatismo materno grave y cirugía múltiple con resultado perinatal exitoso. Revista chilena de obstetricia y ginecología, 76(5), 354-358.

Júnior, G. A. P., Júnior, L. F. H., Atique, J. M. C., Nakamura, E. J., Basile-Filho, A., \& de Andrade, J. I. (1999). Atendimento à gestante traumatizada. Medicina (Ribeirao Preto), 32(3), 282-289.

Labañino, W. L., Muguercia, R. E., Alonso, A. C., Nuñez, E. S., Terrero, I. R., \& Reyes, W. J. (2009). Trauma abdominal en el embarazo. Actualización. Revista Información Científica, 64(4).

Leite, S., Taveira-Gomes, A., \& Sousa, H. (2013). Lesão Visceral em Trauma Abdominal: Um Estudo Retrospetivo. Acta Med Port. 26(6).

Lima, S. O., Cabral, F. L. D., Pinto Neto, A. F., Mesquita, F. N. B., Feitosa, M. F. G., \& Santana, V. R. D. (2012). Avaliação epidemiológica das vítimas de trauma abdominal submetidas ao tratamento cirúrgico. Revista do Colégio Brasileiro de Cirurgiões, 39, 302-306.

Markogiannakis, H., Sanidas, E., Messaris, E., Michalakis, I., Kasotakis, G., Melissas, J., \& Tsiftsis, D. (2006). Management of blunt hepatic and splenic trauma in a Greek level I trauma centre. Acta Chirurgica Belgica, 106(5), 566-571.

Martins-Costa, S. H., Ramos, J. G. L., \& Serrano, Y. L. G. (2005). Trauma na gestação. Revista Brasileira de Ginecologia e Obstetrícia, $27,505-508$. doi.org/10.1590/S0100-72032005000900001.

Mestieri, L. H. M., Domingues, J. R. S., de Oliveira, I. H. L., Bertolli, E., Condi, G. A., \& da Silva Rodrigues, J. M. (2004). Tratamento não-operatório do trauma abdominal fechado. Revista da Faculdade de Ciências Médicas de Sorocaba, 6(2), 4-7.

Mirza, F. G., Devine, P. C., \& Gaddipati, S. (2010). Trauma in pregnancy: a systematic approach. American journal of perinatology, 27(07), 579-586.

Murphy, N. J., \& Quinlan, J. D. (2014). Trauma in pregnancy: assessment, management, and prevention. American family physician, $90(10)$, 717-722.

Parra-Romero, G., Contreras-Cantero, G., Orozco-Guibaldo, D., Domínguez-Estrada, A., \& Bravo-Cuellar, L. (2019). Trauma abdominal: experiencia de 4961 casos en el occidente de México. Cirugía y Cirujanos, 87(2), 183-189.

Ruezga, K. L. L., Gómez, J. A. J., González, L. R. R., Santa Cruz, M. S., Vigna, J. J. G., \& Barba, I. M. T. (2013). Trauma abdominal cerrado y penetrante con lesión a órganos abdominales. Revista Latinoamericana de Cirugía, 3(1), 20-24.

Steenburg, S. D., Padilla-Jones, B., Lee, J.T. et al. (2021). Traumatic injuries of the abdominal wall - a primer for radiologists. Emerg Radiol $28,361-371$. doi.org/10.1007/s10140-020-01842-w

Smith, J., Caldwell, E., D'Amours, S., Jalaludin, B. and Sugrue, M. (2005), Abdominal trauma: a disease in evolution. ANZ Journal of Surgery, $75: 790-794$. https://doi.org/10.1111/j.1445-2197.2005.03524.x

da Silva Silveira, E., \& O’Dwyer, G. (2017). Centro de Trauma: modelo alternativo de atendimento às causas externas no estado do Rio de Janeiro. Saúde em Debate, 41(112), 243-254.

Torres Gómez, D. M. (2020). Características clínicas-epidemiológicas del trauma abdominal en pacientes atendidos en el Servicio de Cirugía del Hospital MINSA II-2 Tarapoto, periodo enero-diciembre 2018. 